\title{
Marktwirtschaftlicher Wettbewerb zur Verbesserung der ambulanten psychiatrischen Versorgung
}

\author{
Economic Competition in the Market to Improve Outpatient Psychiatric Care
}

Autoren

Pro: Hans Joachim Salize

Kontra: Christa Roth-Sackenheim

Bibliografie

DOI 10.1055/s-0028-1090128

Psychiat Prax 2009; 36:

106-109

(c) Georg Thieme Verlag KG

Stuttgart · New York .

ISSN 0303-4259

Korrespondenzadressen

Dr. Christa Roth-Sackenheim

Breite Straße 63

56626 Andernach

c@dr-roth-sackenheim.de

Prof. Dr. Hans Joachim Salize Arbeitsgruppe Versorgungsforschung, Zentralinstitut für Seelische Gesundheit $\mathrm{J} 5$

68159 Mannheim

hans-joachim.salize@

zi-mannheim.de

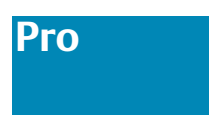

Eines der grundlegenden Theoreme der sogenannten „freien“ Marktwirtschaft besagt, dass die Nachfrage unabhängiger und aufgeklärter Verbraucher das Angebot und damit die Preise der Waren auf dem freien Markt bestimmen. Dass dieser ökonomische Mechanismus im Spätkapitalismus einer weitgehenden Angebotsorientierung gewichen und damit zur Ideologie geworden ist, zeigen u.a. die aufgeblähten PR-Budgets vieler Konzerne, die manchmal sogar höher sind als die eigentlichen Produktionsetats und massiv auf die Weckung künstlicher Bedürfnisse sowie die Ausweitung und Dominanz von Absatzmärkten abzielen - bis hin zum Kollaps dieser Märkte, wie die gegenwärtige Finanzkrise eindrucksvoll belegt.

In dem im Vergleich zum allgemeinen Wirtschaftsleben ungleich stärker staatlich geregelten Gesundheitssektor gilt die Nachfrageorientierung noch viel weniger. Müssten sich die Angebote psychiatrischer Dienste tatsächlich nach der Nachfrage, d.h. den subjektiven Bedürfnissen der Klientel richten, deren Versorgungsvorstellungen und -wünsche sich stark vom fachlich bestimmten Bedarf unterscheiden, würde das viele Einrichtungen der psychiatrischen Versorgung sehr schnell in die Insolvenz treiben. Ein weiterer Effekt wäre vermutlich eine massive Steigerung der Zwangseinweisungsraten.

Aufgrund der charakteristischen Krankheitsmerkmale psychischer Störungen wie kognitive Beeinträchtigungen, Negativsymptomatik, Selbst- und Fremdgefährdung, Wahnsymptome, mangelnde Krankheitseinsicht, defizitäres Hilfesuchverhalten usw. wird die psychiatrische Versorgung immer eine starke angebotsorientierte Komponente aufweisen müssen und damit den Wirkmechanismen eines „freien“ Marktes widersprechen. Es ist ein unumgänglicher und nie endender Prozess, die Steuerungsmechanismen der Inanspruchnahme psychiatrischer Hilfen im- mer wieder neu zu justieren. Neben Wirtschaftlichkeitsfragen werden mit dieser Steuerung auch ethische Fragen nach den Grenzen von Fremdbestimmung in der Versorgung psychisch Kranker aufgeworfen.

Wenn die Preise für psychiatrische Leistungen nicht durch Marktnachfrage frei bestimmt werden können, unterliegen auch sie einer Fremdregelung. Diese Regelungsprozesse sind jedoch nicht notwendig an eine Steuerung der Inanspruchnahme gebunden. Sie sind auch nicht naturwüchsig, wenn man dies angesichts der Festgefügtheit von Finanzierungsverantwortungen und Budgetgrenzen im deutschen Gesundheitswesen auch manchmal zu glauben geneigt ist. Die Teilung der Finanzierungshoheiten in der Versorgung psychisch Kranker in Deutschland zwischen Krankenkassen, kassenärztlicher Vereinigung, überörtlicher Sozialhilfe und Rentenversicherung ist historisch gewachsen. Auch wenn sie aufgrund der langen Tradition unverrückbar wirkt, bleibt diese Aufteilung nichtsdestotrotz künstlich und steht den fachlichen Versorgungsanforderungen einer modernen Psychiatrie entgegen. Die getrennte Finanzierungsverantwortung von stationärer, ambulanter und rehabilitativer Versorgung wurde spätestens mit der Abschaffung der Anstaltspsychiatrie obsolet. Die Psychiatriereform hat jedoch die historische Chance einer grundlegenden Modernisierung der starren und fragmentierten Entgeltsysteme versäumt, obwohl Enthospitalisierung und Flexibilisierung der Versorgungssettings ihr erklärtes Programm war. Somit werden durch die Finanzierungsformen auch 30 Jahre nach der Psychiatriereform weiterhin grundsätzlich falsche Anreize gesetzt und das „anerkannte Übel“ [1] der psychiatrischen Versorgung hierzulande zementiert. Dieses besteht darin, dass eine zeitgemäße Psychiatrie die personenbezogene Sicht- und Vorgehensweise in der Behandlung der Betroffenen notwendig macht, sie aber gezwungen ist, dies 
in einem nach wie vor einrichtungsbezogen strukturierten Versorgungs- und Finanzierungssystem umzusetzen. Alle praktischen Probleme wie separierte Versorgungssektoren, gedoppelte Versorgungsstrukturen, unterbrochene Behandlungsketten, mangelnde Fallsteuerung usw. sind auf diesen grundsätzlichen Widerspruch zurückzuführen.

Alle größeren versorgungspolitischen Initiativen der letzten Jahre arbeiten sich an diesem Problem ab. Dazu zählen die beispielhaft zähen Aktivitäten der Aktion Psychisch Kranke, personenzentrierte Sicht-, Arbeits- und Finanzierungsweisen zu propagieren [2], wie auch die in der Psychiatrie mehr diskutierten als tatsächlich umgesetzten integrierten Versorgungsmodelle. Sie stellen die jüngsten Hoffnungsträger in einer Reihe von Versuchen dar, die Finanzierungsweisen zu flexibilisieren.

Wie jedoch auch die Befunde aus dem Itzehoer integrierten Versorgungsmodell, als dem bisher einzigen, das hierzulande wissenschaftlich begleitet und publiziert wurde [3], zeigen, müssen alle diese Initiativen sich notgedrungen mit sehr bescheidenen Erfolgen zufriedengeben. Das liegt daran, dass sie keine Lösung bieten für die simple Tatsache, dass jeder Einrichtungs- und Finanzierungsträger im fragmentierten System betriebswirtschaftlich agieren muss und im Zweifelsfall die Minderbelastung des eigenen Etats über alle Systemüberlegungen oder übergeordnete Sichtweisen stellt. Der heilige St. Florian („...verschon’ mein Haus, zünd' andere an“) ist und bleibt der Schutzheilige der Finanzierungsträger im deutschen Gesundheitswesen.

Die weitgehende Erfolglosigkeit der bisherigen integrierten Versorgungsmodelle beruht darauf, dass sie viel zu zaghaft, nämlich in der Regel nur die ambulant-ärztliche sowie voll- und teilstationäre Versorgung, integrieren. Gesundheitsökonomische Analysen zeigen aber, dass die Kostenvarianz vor allem bei chronischen psychischen Störungen nur zu rund $50 \%$ durch stationäre, teilstationäre und ambulante ärztlich-psychiatrische Leistungen aufgeklärt wird. Programme, die sich nur auf diese Versorgungssektoren beziehen, erreichen möglicherweise moderate Kostenverschiebungen innerhalb dieser Sektoren, können aber keine Wirkung in den Versorgungsbereichen entfalten, die die andere Hälfte der Kosten verursachen [4,5]. Inwieweit die bisherigen Modelle tatsächlich die ambulante Versorgung psychisch Kranker „verbessern“, bleibt dahingestellt und zudem - aufgrund des Fehlens aussagekräftiger Wirksamkeitsindikatoren - methodisch überaus schwierig nachzuweisen.

Somit ist die gegenwärtige Versorgungsrealität gekennzeichnet von vielen individuellen, regionalen und oft unter großen Eigenbelastungen der Beteiligten ermöglichten Versuchen, die Grenzen des starren Versorgungs- und Entgeltsystems so zu dehnen, damit über Einrichtungs- und Sektorgrenzen hinweg halbwegs sinnvoll kooperiert werden kann. Damit werden die Systemgrenzen jedoch nicht transzendiert. Die Höhe der durch die starren Finanzierungsformen unausgeschöpft bleibenden Synergien ist unbekannt, kann aber als wesentlich vorausgesetzt werden. Was also tun? In einem wie beschrieben strukturierten System einfach nur mehr marktwirtschaftlichen Wettbewerb der Anbieter als Lösung des Dilemmas zu propagieren, wäre nichts weiter als zynischer Neoliberalismus. Auf den starken Mann oder die starke Frau zu warten, der oder die die Finanzierungssysteme dergestalt reformiert, dass sie einer patientenzentrierten Perspektive folgen, ist ebenfalls müßig. Bisher hat sich noch kein Gesundheitsminister diese Aufgabe überhaupt je auf die Fahne geschrieben, weil damit seine Demission von vornherein programmiert wäre. Also bleibt gegenwärtig nur, die Politik der kleinen Schritte weiterzuführen. Diese muss die zarten Liberali- sierungstendenzen der Entgeltsysteme aufgreifen und konsequenter als bisher um- und durchsetzen. Dazu gehört unter anderem Folgendes:

1. Monetäre Anreize wären so zu setzen, dass diejenigen Dienste, die zu einer bedarfsgerechten, aber kostengünstigeren psychiatrischen Behandlung beitragen, auch in den Genuss der erwirtschafteten Einsparungen kommen (die vor allem im stationärpsychiatrischen Sektor anfallen, da Verringerung oder Vermeidung stationärer Aufenthalte der stärkste Hebel zur Kostendämpfung ist). Dies erfordert die Implementierung von Dokumentationssystemen, die diesbezügliche Wirkzusammenhänge ablesen lassen sowie eine wissenschaftliche Begleitforschung von entsprechenden Modellprojekten, mit denen die generierten Nutzeffekte quantifiziert werden.

2. Integrierte Versorgungsmodelle oder Managed-Care-Programme müssen auf Einrichtungen und Dienste des rehabilitativen Sektors ausgeweitet werden, um die bisherigen halbherzigen Ansätze der Flexibilisierung nur der stationärpsychiatrischen und ambulant-fachärztlichen Behandlung auf alle relevanten Versorgungsbereiche zu erweitern.

Diese Maßnahmen sind mit einem nicht unerheblichen organisatorischen, methodischen und politischen Aufwand verbunden. Sie erfordern zudem so etwas wie einen runden Tisch aller relevanten Finanzierungsträger in der Region, die ein entsprechendes Modell wagen will. Die Vorschläge bewegen sich jedoch im Rahmen dessen, was politisch gegenwärtig möglich ist, ohne dass sie das etablierte Entgeltsystem grundlegend infrage stellen. Sie sind gleichbedeutend mit mehr „Markt“ (um die von dieser Debatte geforderte Terminologie aufzugreifen) und wahrscheinlich tendenziell fairerem Wettbewerb der Anbieter. Vor allem die Möglichkeit für Einrichtungsträger, mit guter Versorgung Gewinn zu erwirtschaften, dürfte eine bisher unbekannte Kreativität und damit Synergie entfalten.

Da die Debatte um die Entgeltverfahren der stationärpsychiatrischen Versorgung zurzeit wieder Fahrt aufnimmt und in einigen Teilen Deutschlands ein deutlicher Trend zur Regionalisierung der Versorgung zu beobachten ist (z. B. durch die Kommunalisierung der Eingliederungshilfen in Baden-Württemberg), ist das Klima für innovative Ansätze der Versorgungsfinanzierung gegenwärtig eher günstig. Die Chance sollte nicht vertan werden.

\section{Kontra Laut dem Institut für Qualität und Wirtschaft- lichkeit im Gesundheitswesen IQWIG ist das deutsche Gesundheitssystem grundsätzlich} eines der Besten der Welt. Es ist für den Patienten selbstverständlich, dass wohnortnah, 24 Stunden am Tag, 7 Tage in der Woche und flächendeckend ein ungehinderter Zugang zu medizinischen Leistungen möglich ist. Dabei ist es kaum relevant, ob der Patient in der Eifel oder im Thüringer Wald, in München oder Rostock wohnt. Es macht keinen Unterschied, ob der Patient erwerbstätig, arbeitslos oder berentet ist. Medizinische Leistungen wie z.B. Gelenkersatz werden ohne Berücksichtigung des Alters eines Patienten im Kollektivvertragssystem gewährt. Innovationen finden relativ schnell ihren Weg in die Regelversorgung, so z.B. derzeit die genetischen Untersuchungen oder die MRT-Angiografie. Dabei kann der Patient zu seinem Arzt des Vertrauens gehen, hat also die freie Arztwahl. Seine Beteiligung an der Entscheidungsfindung für seine individuelle Therapie ist politisch erwünscht und wird gefördert. Er kann jederzeit eine zweite Meinung eines anderen Arztes der gleichen oder einer anderen Fachrichtung einholen. 
Es ist in Deutschland undenkbar, dass nur gegen Vorkasse oder sogar gegen diskrete Überreichung eines Geldbetrags im neutralen Umschlag behandelt wird, wie das in manchen europäischen Staaten Alltag ist. Es ist für den deutschen Patienten unvorstellbar, dass es Wartelisten für ambulante und stationäre medizinische Leistungen gibt, die allein aus Kostengründen bestehen. Es kommt in der Alltagserfahrung deutscher Staatsbürger nicht vor, dass der Arzt um die Ecke auf seinem Schild stehen hat: Nur AOK-Patienten. Oder: Nur Teilnahme am $73 \mathrm{~b}$-Vertrag der GEK, nur Versicherte mit Teilnahme am DMP Diabetes der BKK Mobil OIL, der BKK Essanelle und der Techniker Krankenkasse in Nordrhein, Bezirk Aachen. Oder dass er in ein Krankenhaus muss, das für seine Familie nur nach einer Tagesreise erreichbar ist, da seine Krankenkasse zum Beispiel nur mit einer bestimmten Klinikkette wie Helios, Rhön-Klinikum oder Asklepios einen Vertrag hat.

Die Frage ist aber: Wie steht es mit diesen, uns durchaus selbstverständlich gewordenen, aber dennoch hochgeschätzten Werten wie der Flächendeckung, der „Rund-um-die-Uhr“-Versorgung sowie der freien Arztwahl und dem Zugang zu Innovationen, wenn sich die Gesetze der freien Marktwirtschaft und des politisch gewollten Wettbewerbs im Gesundheitswesen durchsetzen? Wie steht es dann mit der Sicherheit und der Gerechtigkeit?

Und weiter: Was bedeutet das für uns Ärzte, wenn wir unsere Leistungen selbst betriebswirtschaftlich kalkulieren und mit den einzelnen Kassen in Verhandlungen treten müssen? Wenn diese Verträge mit den Kassen lediglich für einen bestimmten Zeitraum gelten und danach neu verhandelt werden müssen? „Wettbewerb folgt nicht den Kriterien einer flächendeckenden, qualitativ hochwertigen Versorgung für alle mit freier Arztwahl und niedrigschwelligem Zugang zu den Leistungen. Wettbewerb funktioniert auch im Gesundheitswesen nach den Kriterien des Marktes, nach Angebot und Nachfrage und damit auch nach der Zahlungsbereitschaft und Zahlungsfähigkeit der potenziellen Kunden. Renditen sind wichtiger als Werte wie Solidarität und Schutz von Benachteiligten. Das führt zu Mehrklassenmedizin, zu Zugangshindernissen, zur Benachteiligung bestimmter Patientengruppen..." (Dr. Andreas Köhler, Vorstandsvorsitzender der Kassenärztlichen Bundesvereinigung, in seiner Rede vor der öffentlichen Vertreterversammlung der KBV am 5.12.08 in Berlin).

Welche Patientengruppen werden das wohl sein, die hier von Dr. Köhler gemeint sind? Machen wir uns nichts vor: Es werden die Patientengruppen sein, die am meisten von struktureller Stigmatisierung betroffen sind, und das sind nun mal unsere Patienten mit chronischen und schweren psychischen und z.T. auch neurologischen Erkrankungen. Die Stigmatisierung nimmt zu, je mehr die Erkrankung nach außen sichtbar wird, je mehr das Verhalten und die sozialen Beziehungen des Patienten durch die psychische Erkrankung negativ beeinflusst werden. Dies führt bekanntlich auch zu wirtschaftlichen Nachteilen bei Menschen mit psychischen Erkrankungen, da sie häufig gar nicht erst in einen Beruf gekommen, frühberentet oder in Einrichtungen untergebracht sind, die nach den Gesetzen des SGB XI über die Pflegeversicherung finanziert werden.

Seit Langem werden psychisch kranke Menschen schon als teure Patienten mit schlechten Risiken z.B. bei der Aufnahme in die private Krankenversicherung, die Berufsunfähigkeits-, Krankenhaustagegeld- oder Krankengeldversicherung systematisch ausgegrenzt. Der Gesetzgeber hat nun für die gesetzliche Krankenversicherung ab 2009 in der Konstruktion des Gesundheitsfonds mittels des morbiditätsbedingten Risikostrukturausgleiches („Morbi-RSA“) versucht, einer wettbewerbsbedingten Bevorzugung junger gesunder Menschen entgegenzuwirken, indem er Krankenkassen, die chronisch Kranke versichern, mit einem Geldzuschlag pro Patient belohnt. Insgesamt 80 Krankheiten sind benannt, die einen solchen Zuschlag auslösen. Unter diesen 80 Genannten befinden sich 24 Krankheiten, die das Gehirn betreffen, davon wiederum sind 8 psychische Erkrankungen: Delir, Demenz, schwerwiegender Alkohol- und Drogenmissbrauch, psychotische Störungen und Persönlichkeitsstörungen, Depression, bipolare affektive Störungen, Anorexia und Bulimia nervosa, Aufmerksamkeitsstörungen.

Aber wird das die ambulante psychiatrische Versorgung besser stellen? Einerseits ist ein Anreiz für die gesetzlichen Krankenkassen geschaffen, möglichst viele Versicherte mit einer dieser 80 Diagnosen zu „versehen“, da sich dadurch ein größeres Stück am Gesamtversorgungs-,,Kuchen“ einheimsen lässt. Andererseits besteht ein großer Anreiz für die Kasse, die Kosten für diese Morbi-RSA-Versichertengruppe zu drücken. Da der Gesundheitsfond nachweislich durch den Krankenversicherungs-Einheitsbeitrag von 15,5\% um mindestens 0,3 Prozentpunkte unterfinanziert ist (das bedeutet 3 Milliarden Euro) und die Auswirkungen der Finanzkrise auf die steuerlichen Geldflüsse in den Fonds noch unabsehbar sind, kann die Krankenkasse nur profitieren, wenn sie möglichst viele Menschen mit Morbi-RSA-Diagnosen versichert hat aber möglichst wenig für diese ausgibt. Dies wird umso fataler, je niedriger die jährliche Anpassung des Gesundheitsfonds ausfällt. Die Ausgaben für Gesundheit sind in Zukunft noch direkter als bisher abhängig von der wirtschaftlichen Lage des Staates. Außerdem werden die Zuschläge sinken müssen, je mehr Versicherte an den betreffenden Diagnosen erkranken.

Für die ambulante psychiatrische Versorgung bedeutet dies, dass um die Ressourcen künftig noch härter gekämpft werden muss. Während andere medizinische Fachgebiete, wie z.B. die Innere Medizin, sich in der Blüte ihres wissenschaftlichen Erkenntniszuwachses während der Zeiten der Vollbeschäftigung in der BRD in einer sehr guten finanziellen Ausstattung des Gesundheitswesens entwickeln und Ressourcen für sich beanspruchen konnten, fiel der Aufschwung der Psychiatrie und Psychotherapie bereits in eine Zeit steter gesundheitspolitisch bedingter Ressourcenverknappung. Wir haben weiter einen Anstieg der Morbidität bei psychischen Störungen zu erwarten - wegen der Zunahme degenerativer Erkrankungen des Gehirns und wegen vermehrter Inanspruchnahme vor allem bei depressiven Störungen.

Dennoch soll nicht außer Acht gelassen werden, dass die Ausgaben für die ambulante Versorgung insgesamt Jahr für Jahr steigen. Aber trifft das auch für die ambulante psychiatrische Behandlung zu? Hier sind Zweifel angebracht. Die sog. „Melchinger-Studie“ 2008 im Auftrag der Kassenärztlichen Bundesvereinigung zeigte, dass wie bereits vor 5 Jahren die meisten Ressourcen für psychische Erkrankungen im Bereich der Richtlinienpsychotherapie ausgegeben werden. Im Klartext: Für rund 75\% der psychisch kranken Menschen in der ambulanten psychiatrischen Regelversorgung wird nur ein Viertel der Gesamtausgaben für psychische Störungen aufgewandt. Umgekehrt fließen $75 \%$ der Gesamtausgaben für alle psychischen Störungen in Deutschland in die Vergütung der Richtlinienpsychotherapie, die rund 25\% der Patienten zugutekommt. Das heißt: Die Versorgung mit Richtlinienpsychotherapie ist sicherlich verbessert worden. Diese kommt aber den schwer chronisch Erkrankten 
nicht zugute, sondern eher den Patienten, die durch diese Verfahren gut erreichbar sind, sie für sich nutzen können und hierdurch allein ein Verbesserungspotenzial haben. Die Versorgung schwerer psychisch Kranker, die einer lebenslangen, krankheitsstadienspezifischen und komplexen Behandlung bedürfen, hat sich jedoch weiter verschlechtert.

Das IGES-Gutachten zur ambulanten neuropsychiatrischen Versorgung vom Oktober 2007 zeigt, dass pro Jahr in der gesetzlichen Krankenversicherung außerhalb der Richtlinienpsychotherapie für einen psychisch kranken Menschen immer weniger Geld aufgewandt wird. Nervenärzte und Psychiater sind die Fachgruppe, die trotz Einführung der Praxisgebühr im Gegensatz zu allen anderen Fachgruppen steigende Fallzahlen zu verzeichnen haben.

FAZIT: Es ist zu befürchten, dass marktwirtschaftlicher Wettbewerb die ambulante psychiatrische Versorgung eher noch weiter verschlechtern wird.

\section{Literatur}

1 Schmidt-Michel P, Kuhn F, Bergmann F. Die integrierte Versorgung per Gesetz ist für die Psychiatrie gescheitert. Psychiat Prax 2008; 35: 57-59

2 Kunze H. Personenbezogene Behandlung in psychiatrischen Kliniken und darüber hinaus - Gute Praxis und Ökonomie verbinden. Psychiat Prax 2007; 34: 145-154

3 Roick C, Heinrich S, Deister A et al. Das Regionale Psychiatriebudget: Kosten und Effekte eines neuen sektorübergreifenden Finanzierungsmodells für die psychiatrische Versorgung. Psychiat Prax 2008; 35: 279-285

4 Kilian R, Angermeyer M. Der Einfluss der Neuroleptikabehandlung auf die Inzidenz und die Kosten stationärer psychiatrischer Behandlungen bei schizophren Erkrankten: Ergebnisse einer prospektiven Beobachtungsstudie. Psychiat Prax 2004; 31: 138-146

5 Salize HJ. Die Kosten der Schizophrenie - Was wissen wir (nicht)? Psychiat Prax 2001; 28: S21-S28

6 Melchinger H. Umsteuerungen dringend geboten. Dtsch Ärztebl 2008; 105 (46): A2457-2460

7 Melchinger H, Machleidt W, Rössler N. Psychiatrische Versorgung: Ausgaben auf dem Prüfstand. Dtsch Ärztebl 2003; 100 (44): A2850-2852

8 Strukturen und Finanzierung der ambulanten neurologischen und psychiatrischen Versorgung, Institut für Gesundheits- und Sozialforschung IGES, Wichmannstraße 5, 10787 Berlin, Oktober 2007

9 IQWIG. German health care: a bit of Bismarck plus more science. British Medical Journal 2008; 337: 1142-1145 